\title{
Recurrence of a de-differentiated liposarcoma of the colon
}

\author{
Mairead M. Hennessy, John E. O'Connell, David E. Kearney, Emmet J. Andrews \\ Department of Surgery, Cork University Hospital, Wilton, Cork, Republic of Ireland
}

Received: March 9, 2017

Accepted: May 3, 2017

Online Published: May 15, 2017

DOI: $10.5430 /$ crcp.v4n2p24

URL: https://doi.org/10.5430/crcp.v4n2p24

\begin{abstract}
We present the case of a 56-year-old gentleman who presented with non specific malaise, myalgia and anorexia. Clinical examination revealed a large circumscribed palpable mass in the left iliac fossa. He underwent an intraabdominal pelvic biopsy, computed tomography (CT), magnetic resonance imaging (MRI) of abdominal wall and Positron Emmission Tomography (PET). Differential diagnoses included Gastro Intestinal Stromal Tumour (GIST) and sarcoma. He underwent a laparotomy and excision of the lesion. Histological analysis of the lesion demonstrated a dedifferentiated liposarcoma involving the colon and adjacent pericolic fat. Margins were clear and there was no nodal involvement. He declined adjuvant chemotherapy. He subsequently developed a local recurrence, which was deemed unresectable. He commenced chemotherapy but continued to deteriorate. He received palliative treatment and died 6 months following his initial surgery.
\end{abstract}

Key Words: Liposarcoma, Sigmoid colon, De-differentiated liposarcoma, Recurrence

\section{INTRODUCTION}

Liposarcoma, the most common soft tissue sarcoma, represents $20 \%$ of the mesenchymal malignancies, most commonly occurring in the retropertioneum and extremities. Colonic liposarcoma is extremely rare.

Several cases of dedifferentiated liposarcoma of the small bowel mesentery have been described. However, the number of dedifferentiated liposarcoma of the sigmoid mesocolon is few. Some pathologists do acknowledge that a number of intra-abdominal poorly differentiated sarcoma are dedifferentiated liposarcomas.

To our knowledge, this is the sixth case of primary liposarcoma of the sigmoid colon reported in the literature.

\section{CASe PResentation}

A 56-year-old gentleman presented with non specific malaise, myalgia and anorexia. Past medical history included mitral valve endocarditis for which he underwent a mitral valvuloplasty 7 years previously. He also suffered from gout. Clinical examination revealed a large circumscribed, palpable mass in the left iliac fossa. The remainder of the clinical examination was normal. Laboratory investigations showed; haemoglobin $12.1 \mathrm{~g} / \mathrm{dl}$, white cell count $8.8 \times 10^{9}$, C Reactive Protein $200 \mathrm{mg} / \mathrm{L}$ and ESR $118 \mathrm{~mm} / \mathrm{hr}$.

Computed tomography (CT) thorax, abdomen and pelvis revealed a large heterogeneously enhancing intraperitoneal left iliac fossa mass with a peri-lesional $1.2 \mathrm{~cm}$ node (see Figure 1). MRI abdomen again identified the large mesenteric mass.

*Correspondence: Mairead M. Hennessy; Email: mhennessy@muh.ie; Address: Department of Surgery, Mercy University Hospital, Grenville Place, Cork, Republic of Ireland. 
It appeared intimately related to an adjacent loop of descending colon, of normal calibre and a loop of ileum which was thickened and likely involved. A positron emission tomography (PET) showed a hypermetabolic large lower abdominal mass (see Figure 2). An intraabdominal pelvic mass biopsy was not diagnostic.

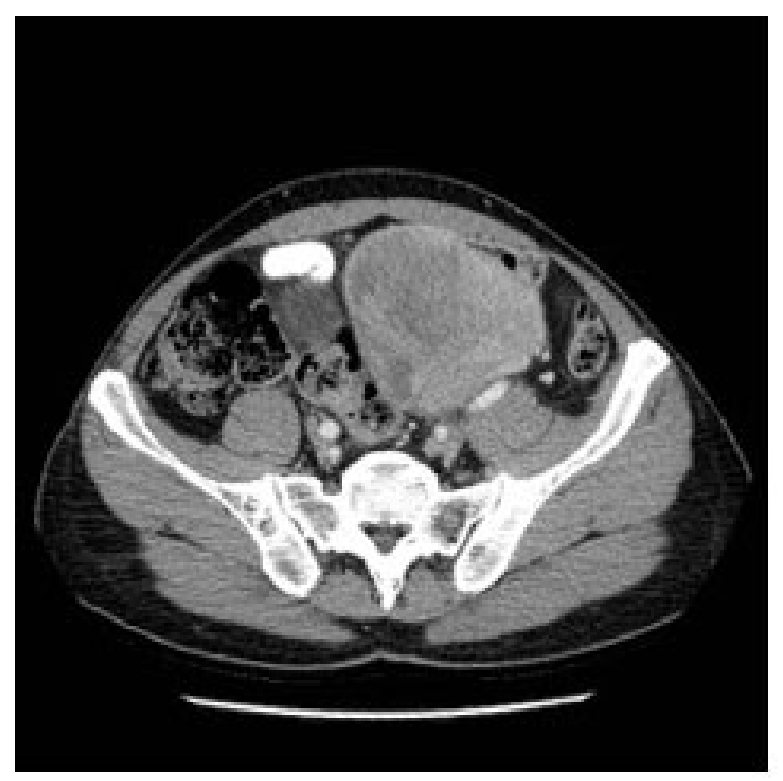

Figure 1. CT demonstrating tumour of sigmoid colon

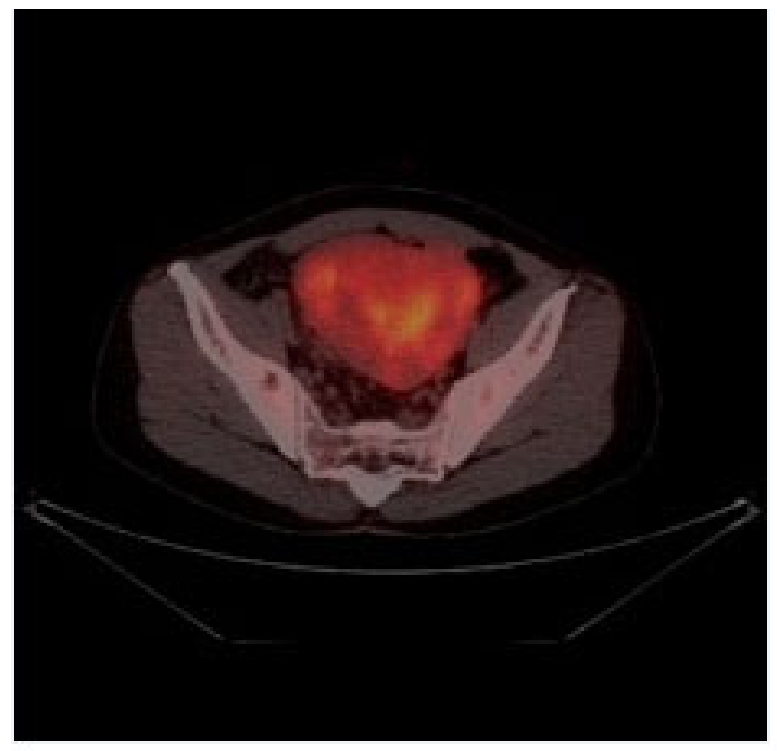

Figure 2. PET CT demonstrating hypermetabolic tumour

Following discussion at the multidisciplinary team (MDT) meeting, he underwent a lower midline laparotomy. This identified an ovoid tumour from the antimesenteric border of mid-sigmoid (involving mid sigmoid $10 \mathrm{~cm}$ ) and adherent to the dome of the bladder. No liver or peritoneal metasPublished by Sciedu Press tases were visible. The mass was mobilized and the dome of the bladder/peritoneum excised. The bladder wall was not breached. The left colon was mobilized and upper rectum/distal sigmoid transected with a GIA 80; ligasure to the mesentry. The distal descending colon was transected with a GIA 80 and good pulsatile bleeding from the marginal artery was observed. An end to side anastomosis was performed. The lesion was sent for histo-pathological analysis. He had an uneventful post-operative course and was discharged home 6 days post operatively.

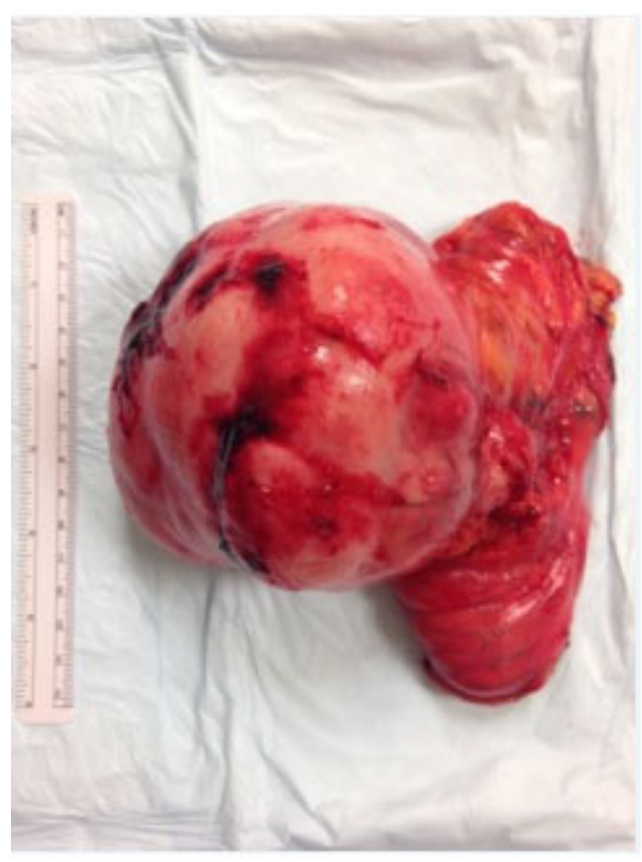

Figure 3. Liposarcoma of the sigmoid colon specimen

Macroscopic examination of the specimen showed a large, oval, solid, cream coloured tumour measuring $9.5 \mathrm{~cm} \times$ $3 \mathrm{~cm} \times 3 \mathrm{~cm}$ (see Figure 3 ). The mucosal surface appeared flattened with the tumour overlying it. The remainder of the mucosal surface appeared normal. The tumour did not invade through the mucosa. Sectioning of the tumour identified a predominantly solid cream white homogenous cut surface with some areas of degeneration and possible necrosis throughout the tumour.

Microscopic examination revealed a spindle cell tumour with the cells arranged in a storiform pattern. Numerous pleomorphic cells with some tumour giant cells and numerous mitoses, many abnormal, were present (see Figure 4). Immunohistochemistry of the tumours cells only stained positively for vimentin and very focal weak staining for CD117, probably of no significance. Additional blocks of the tumour were sampled, particularly from the pericolic fat and occasional lipoblasts were identified. The tumour involved the 
submucosa, focally extending into the mucosa, muscularis propria and there were several deposits within the pericolic fat. The distal and proximal margins were free of tumour and nine lymph nodes were free of tumour.

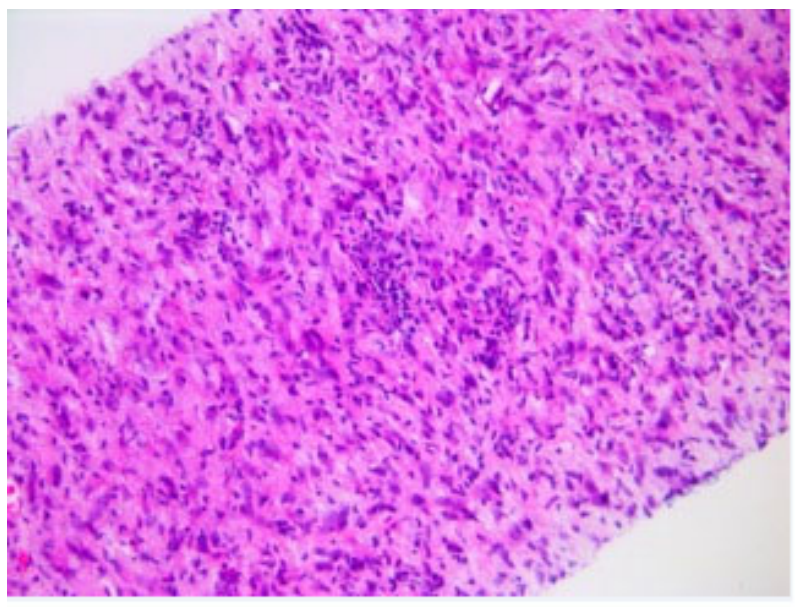

Figure 4. Histological slide demonstrating spindle cell tumour with the cells arranged in a storiform pattern

The histopathological diagnosis was of a dedifferentiated liposarcoma involving the colon and adjacent peri-colic fat, $\mathrm{R} 0$ resection. However, in view of behaviour of the tumour, residual disease could not be excluded.

Post operatively, the case was again discussed at the MDT meeting. By consensus, it was decided the patient should be offered adjuvant chemotherapy, but its benefit was unclear. The patient declined adjuvant treatment.

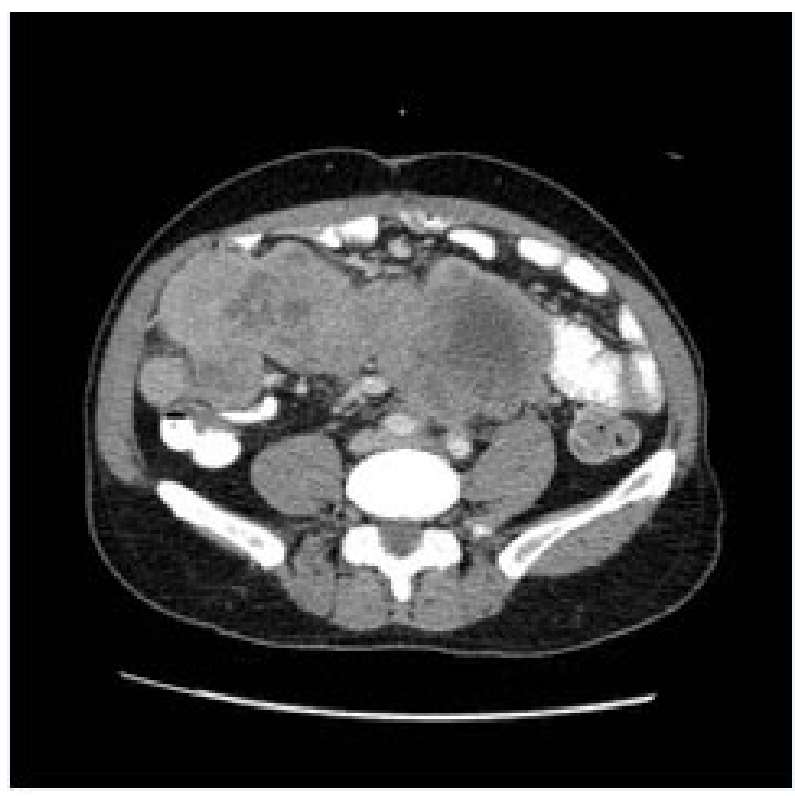

Figure 5. CT showing tumour recurrence
He represented three months post operatively with weight loss and night sweats. A CT thorax, abdomen and pelvis demonstrated a large volume local recurrence with a large lobulated abdomino-pelvic mass lesion with compressive effects (see Figure 5). Again, the case was discussed at the MDT meeting. The disease was not suitable for surgical resection. He commenced chemotherapy but continued to deteriorate and died one month later.

\section{Discussion}

Liposarcoma is a frequently occurring soft tissue tumour found in adults, usually in the retroperitoneum and the extremities. It is uncommonly found in the colon. The first case of primary liposarcoma of the colon was reported by Wood and Morgenstern in 1989. ${ }^{[1]}$

In February 2013, the World Health Organization (WHO) published "Classification of Tumours of Soft Tissue and Bone". This provided reproducible diagnostic criteria for pathologists in addition to an updated classification system. In recent years, there have been huge advances made in immunohistochemical, genetic and molecular testing. These guidelines are important for practicing surgeons, pathologists and oncologists.

The 2013 WHO classification includes several new categories (pseudomyogenic haemangioendothelioma, haemosiderotic fibrolipomatous tumour and acral fibromyxoma), gastrointestinal stromal tumours, nerve sheath tumours, and undifferentiated soft tissue tumours. New genetic and molecular data for established tumour types were included as they facilitated improved pathological definition and diagnosis. ${ }^{[2]}$

Interestingly, tumours previously classified as "mixed- type liposarcoma" are now considered unusual examples of dedifferentiated liposarcoma.

MDM2 and CDK4 are immunohistochemical markers used to diagnose atypical lipomatous tumour, well-differentiated liposarcoma and dedifferentiated liposarcoma. They are antibodies against the gene products coded on 12q13-15 that are augmented in these tumour types. ${ }^{[3,4]}$ For negative or equivocal immunohistochemical results found in clinically suspicious lesions, molecular studies (frequently FISH) are indispensable.

Retention of the term "well-differentiated liposarcoma" is appropriate for tumours in sites where margin-negative resection is not possible (e.g., retroperitoneum, mediastinum). These tumours are associated with significant mortality. ${ }^{[2]}$

Dedifferentiated liposarcoma is known to exhibit "homologous lipoblastic differentiation" in some cases. The dedifferentiated component exhibit lipoblasts which have morpho- 
logical features indistinguishable from pleomorphic liposarcoma. ${ }^{[5]}$

Primary liposarcoma of the colon tends to occur in adults with a peak incidence between 5th and 6th decades, with a slender male predominance. Most tumours (75\%) are located in the right colon. Symptoms associated with colonic or retroperitoneal liposacrcoma are non-specific and vague. ${ }^{[1,6,7]}$ Clinical examination may reveal a palpable abdominal mass. Patients frequently present at a late stage. Sato et al. reported a case where melena was the presenting complaint $^{[8]}$ while Choi et al. described a case of a liposarcoma of the ascending colon in 2011 which presented as haemoperitoneum. ${ }^{[9]}$ Gutsu et al. described the first case report of an extraluminal mass. ${ }^{[10]}$ Mixed-type colonic liposarcoma and well differentiated retroperitoneal liposarcoma were confirmed in the pathology report. Their particular patient remained disease free at 24 months following surgical resection. ${ }^{[11]}$

Similarly, D'Annibale et al. reported a case of primary liposarcoma of the colon presenting as a massive intraluminal lesion. They concluded that as no guidelines are available for the management of liposarcoma of the colon, surgical resection should be performed when feasible. ${ }^{[12]}$

Unfortunately only a few cases of colonic liposarcoma have been published and so an ideal diagnostic guideline has not yet been established. Both CT and MR are valuable but accurate preoperative diagnosis is not possible in all cases. Only histopathologic examination can confirm the diagnosis. All radiological investigations are completed prior to undergoing colorectal surgery. Suzuki et al. found that the radiology reflected the pathological findings accurately. ${ }^{[11]}$

Surgical resection is the treatment of choice. ${ }^{[13]}$ An R0 resection is the best predictor of overall survival. In achieving this, patients often require resection of the adjacent organ, most commonly kidney $32 \%$ and colon $25 \%$. Local recurrence is common; as high as $41 \%$ for retroperitoneal liposarcoma. ${ }^{[13]}$ In our case, both the sigmoid colon and the bladder were involved. Fortunately, invasion in to other organs was not evident.

A similar case to this was published in November 2013 where a patient underwent laparotomy for excision of intraperitoneal dedifferentiated liposarcoma. The patient did not receive adjuvant treatment. Five months post laparotomy, the patient re-presented with a locoregional recurrence. ${ }^{[14]}$

Dedifferentiated liposarcoma has a worse prognosis than other subtypes because it has a higher risk of local recurrence and distant metastasis ${ }^{[15]}$ and also because the recurrent tumours tend to dedifferentiate over time. ${ }^{[13]}$ It therefore has higher malignant potential when compared to other subtypes. Reflecting this malignant potential, dedifferentiation of well-differentiated liposarcomas may occur when a tumour infiltrates other organs when recurrence ensues.

In conclusion, we report a case of liposarcoma of the sigmoid colon. In spite of meticulous surgical technique, achieving an R0 resection and close follow-up, fatal recurrence occurred. As the numbers of cases increases, guidelines for the treatment and management of these rare tumours will be established.

\section{Learning points}

- Although liposarcomas of the colon are rare, they should always be within your differential diagnosis. MDT discussion prior to surgery is essential.

- Histopathological analysis of the tumour is always required to confirm the suspected diagnosis.

- Currently, surgical excision of the tumour is the gold standard of management and treatment. However, surgeons should be cognisant of the tendancy for these lesions to recur despite achieving clear margins at surgery.

- Given the tendancy for recurrence, oncological advise must be sought early to determine if adjuvant treatment should be administered.

\section{Conflicts of InTEREST Disclosure}

None.

\section{REFERENCES}

[1] Wood DL, Morgenstern L. Liposarcoma of the ileocecal valve: a case report. Mt Sinai J Med. 1989; 56(1): 62-4. PMid: 2784186.

[2] Fletcher CD. The evolving classification of soft tissue tumours - an update based on the new 2013 WHO classification. Histopathology. 2014; 64(1): 2-11. PMid: 24164390. https://doi.org/10.111 $1 /$ his. 12267

[3] Binh MB, Sastre-Garau X, Guillou L, et al. MDM2 and CDK4 im- munostainings are useful adjuncts in diagnosing well-differentiated and dedifferentiated liposarcoma subtypes: a comparative analysis of 559 soft tissue neoplasms with genetic data. Am J Surg Pathol. 2005; 29(10): 1340-7. PMid: 16160477. https://doi.org/10.1097/ 01.pas. 0000170343.09562 .39

[4] Sirvent N, Coindre JM, Maire G, et al. Detection of MDM2-CDK4 amplification by fluorescence in situ hybridization in 200 paraffinembedded tumor samples: utility in diagnosing adipocytic lesions 
and comparison with immunohistochemistry and real-time PCR. Am J Surg Pathol. 2007; 31(10): 1476-89. PMid: 17895748. https: //doi.org/10.1097/PAS.0b013e3180581fff

[5] Mari-o-Enríquez A, Fletcher CD, Dal Cin P, et al. Dedifferentiated liposarcoma with "homologous" lipoblastic (pleomorphic liposarcomalike) differentiation: clinicopathologic and molecular analysis of a series suggesting revised diagnostic criteria. Am J Surg Pathol. 2010; 34(8): 1122-31. PMid: 20588177. https://doi.org/10.1097/ PAS.0b013e3181e 5dc49

[6] Parks RW, Mullan FJ, Kamel HM, et al. Liposarcoma of the colon. Ulster Med J. 1994; 63(1): 111-3. PMid: 8658985.

[7] Chen KT. Liposarcoma of the colon: a case report. Int J Surg Pathol. 2004; 12(3): 281-5. PMid: 15306943. https://doi.org/10.117 $7 / 106689690401200312$

[8] Sato Y, Yamamoto S, Fujita S. Retroperitoneal liposarcoma with colonic involvement: a case report. Jpn J Clin Oncol. 2014; 44(4): 374-8. PMid: 24558126. https ://doi.org/10.1093/jjco/hyu 009

[9] Choi YY, Kim YJ, Jin SY. Primary liposarcoma of the ascending colon: a rare case of mixed type presenting as hemoperitoneum combined with other type of retroperitoneal liposarcoma. BMC Cancer. 2010; 10: 239. PMid: 20507577. https://doi.org/10.1186/14 71-2407-10-239
[10] Gutsu E, Ghidirim G, Gagauz I, et al. Liposarcoma of the colon: a case report and review of literature. J Gastrointest Surg. 2006; 10(5): 652-6. PMid: 16773759. https://doi.org/10.1016/j.gassur .2005 .09 .014

[11] Suzuki S, Hirasaki S, Yumoto E, et al. A case of liposarcoma of the ascending colon mesenterium. Nihon Shokakibyo Gakkai Zasshi. 2009; 106(9): 1343-50. PMid: 19734706.

[12] D'Annibale M, Cosimelli M, Covello R, et al. Liposarcoma of the colon presenting as an endoluminal mass. World J Surg Oncol. 2009; 7: 78. PMid: 19852822. https://doi.org/10.1186/1477-781 9-7-78

[13] Neuhaus SJ, Barry P, Clark MA, et al. Surgical management of primary and recurrent retroperitoneal liposarcoma. Br J Surg. 2005; 92(2): 246-52. PMid: 15505870. https://doi.org/10.1002/bj s. 4802

[14] Grifasi C, Calogero A, Carlomagno N, et al. Intraperitoneal dedifferentiated liposarcoma showing MDM2 amplification: case report. World J Surg Oncol. 2013; 11: 305. PMid: 24279301. https : //doi.org/10.1186/1477-7819-11-305

[15] Singer S, Antonescu CR, Riedel E, et al. Histologic subtype and margin of resection predict pattern of recurrence and survival for retroperitoneal liposarcoma. Ann Surg. 2003; 238(3): 358-70; discussion 70-1. https://doi.org/10.1097/01.sla.000008654 2.11899 .38 